\title{
Correction to: Postoperative atrial fibrillation is less frequent in pulmonary segmentectomy compared with lobectomy
}

\author{
Takuya Ueda $^{1} \cdot$ Kenji Suzuki $^{1} \cdot$ Takeshi Matsunaga $^{1} \cdot$ Kazuya Takamochi $^{1} \cdot$ Shiaki Oh $^{1}$
}

Published online: 5 December 2017

๑) The Japanese Association for Thoracic Surgery 2017

\section{Correction to: General Thoracic and Cardiovascular Surgery https://doi.org/10.1007/s11748-017-0858-x}

In the original publication of this article, the Table 1 was published incorrectly with missing data.

The revised Table 1 is given in the following page. The original article was corrected.

The original article can be found online at https://doi. org/10.1007/s11748-017-0858-x.

Kenji Suzuki

kjsuzuki@juntendo.ac.jp

Takuya Ueda

t-ueda@juntendo.ac.jp

Takeshi Matsunaga

matsu812@juntendo.ac.jp

Kazuya Takamochi

ktakamo@juntendo.ac.jp

Shiaki Oh

toh@juntendo.ac.jp

1 Department of General Thoracic Surgery, Juntendo

University School of Medicine, 1-3 Hongo 3-chome,

Bunkyo-ku, Tokyo 113-8431, Japan 
Table 1 Clinicopathological features of the lobectomy/ segmentectomy groups

\begin{tabular}{|c|c|c|c|}
\hline Variable & Lobectomy $(n=443)$ & Segmentectomy $(n=164)$ & $p$ value $^{\mathrm{a}}$ \\
\hline \multicolumn{4}{|l|}{ Gender } \\
\hline Male & $226(51.0 \%)$ & $68(41.5 \%)$ & 0.04 \\
\hline \multicolumn{4}{|l|}{ Age } \\
\hline Years (median) & $67(35-86)$ & $67(27-84)$ & 0.17 \\
\hline \multicolumn{4}{|l|}{ BMI } \\
\hline Less than 18.5 & $33(7.5 \%)$ & $9(5.5 \%)$ & 0.40 \\
\hline More than 25 & $107(24.2 \%)$ & $36(22.0 \%)$ & 0.57 \\
\hline \multicolumn{4}{|l|}{ Smoking status (pack-year) } \\
\hline 40 or more & $290(23.9 \%)$ & $26(15.9 \%)$ & 0.03 \\
\hline \multicolumn{4}{|l|}{ Preoperative pulmonary function } \\
\hline \multicolumn{4}{|l|}{ FEV $1.0 \%$} \\
\hline Less than $70 \%$ & $106(23.9 \%)$ & $30(18.3 \%)$ & 0.17 \\
\hline \multicolumn{4}{|l|}{$\% \mathrm{VC}$} \\
\hline Less than $80 \%$ & $44(10.0 \%)$ & $17(10.6 \%)$ & 0.83 \\
\hline History of ischemic heart disease & $42(9.5 \%)$ & $11(6.7 \%)$ & 0.28 \\
\hline History of lung cancer resection & $12(2.7 \%)$ & $24(14.6 \%)$ & $<0.01$ \\
\hline \multicolumn{4}{|l|}{ Operation time } \\
\hline Minutes (median) & $144(35-352)$ & $156(61-315)$ & $<0.01$ \\
\hline \multicolumn{4}{|l|}{ Blood loss } \\
\hline ml (median) & $20(0-605)$ & $15(0-410)$ & 0.09 \\
\hline Mediastinal LN dissection & $313(70.7 \%)$ & $48(29.3 \%)$ & 0.01 \\
\hline POAF & $34(7.7 \%)$ & $3(1.8 \%)$ & 0.01 \\
\hline
\end{tabular}

$B M I$ body mass index, FEV1.0\% forced expiratory volume $\%$ in one second, $\% V C \%$ volume capacity, $L N$ lymph node, $P O A F$ postoperative atrial fibrillation

${ }^{a}$ Logistic regression analysis 